\title{
ADAPTIVE BEAMFORMER WITH COMBINATION OF SUBBAND FILTERING FOR HEARING-AID SYSTEMS BACKGROUND NOISE REDUCTION
}

\author{
Cheng-Chi Tal, Chih-Hsing Chang, Chuan-Ching Tan, Tsung-Wen Huang, Ching-Chau Su \\ Department of Electrical Engineering, National Cheng Kung University, \\ Tainan, Taiwan
}

\begin{abstract}
In this paper, we present a noise reduction technique for hearing-aid systems. The proposed algorithm adopted adaptive beamformer with combination of subband filtering technique. The structure of conventional hearing aids is relatively simple. They amplify ambient sounds that include speech signal as well as noise. Because noise and human speech signal are amplified at the same time, hearing-aid users can't clearly hear speech signal in noisy environment. The direction of sound can be used to discriminate speech signal from noise by combining adaptive noise canceller and adaptive beamformer. We have developed a system that based on the constrained adaptive noise canceller to preserve speech signal from straight ahead and minimize background noise arriving from other directions. This system also uses subband filtering technique to reduce the requirement for computation and enhance the flexibility of the system. The performance of this system is illustrated using simulated and real-world noises. The results show that the developed system can reserve the right ahead speech signal and substantially reject noises from other directions.
\end{abstract}

Biomed Eng Appl Basis Comm, 2002 (April): 14: 55-66.

Keywords : Hearing aids, Subband adaptive filtering, Beamformer

\section{INTRODUCTION}

Traditional hearing aids amplify all sounds without discriminating between the desired speech and the background noises. Hearing-aid users are always harassed with the background noises. For example, in a noisy factory, when a hearing-impaired listener who wears a traditional hearing aid wants to talk to other people, both speech signals and noises will be amplified at the same time. In such a circumstance, a person still can't communicate with others, and his or her hearing might be damaged even more by loud noises.

Reccived: April 2, 2002; Accepted: April 10, 2002 Correspondence: Cheng-Chi Tai, professor

Department of Electrical Engineering Naional Cheng Kung University 1 University Road, Tainan, Taiwan E-mail: ctai@mail.ncku.edu.tw
The direction of sound can be used to discriminate speech signal from noise by combining adaptive noise canceller [ $1-3]$ and adaptive beamformer [4]. To apply adaptive beamforming technique, it requires at least two microphones. So the hearing aids must be binaural type. Usually, the direction of the speech signal is fixed and often in front of user. When the speech signal sources move into the effective range of the hearing aids, the hearing aids can track the source and enhance signal-to-noise ratio (SNR) of the speech signal until the speech signal sources move out of the effective range. And further, it must have the automatic gain control to avoid too loud signal which will hurt user's hearing.

Adaptive filter algorithms are usually used for the achievement of noise cancellation. Owing to the requirement of high-order coefficients for the adaptive filter when applied to the noise cancellation system, the processing system require vast computation and hence reduce the convergent speed. Subband adaptive 
filter technique can reduce the requirements for computation and storage space. Basically, subband adaptive filter algorithm $[5-8]$ is constructed with both multirate filter banks $[9,10]$ and adaptive filter algorithms. Since human perception of audio signals is frequency dependent, subband adaptive filtering also allows nonuniform frequency resolution that leads to the use of different decimators for different bandwidths [11-13].

The noise cancellation system we developed is based on Griffiths-Jim beamformer with combination of subband filtering to provide directional noise cancellation capability. We developed a MATLAB ${ }^{\text {TM }}$ program to verify the noise cancellation capabilities of the algorithm. We also implemented the real-time system for the binaural type hearing aids using a Texas Instruments TMS320VC5402 digital signal processor development starter kit (DSK).

In the following sections, we will briefly review the adaptive beamforming and subband adaptive filtering techniques. Hardware system and software are described in Section 3. Experiments and results are given in Section 4. Finally, we conclude with a discussion in Section 5.

\section{THEORETICAL BACKGROUND}

\subsection{Array processing and Griffiths-Jim beam- former}

The basic idea of array processing is to collect the variation of signals in the environment using multiple sensors. When there is more than one sensor, the amplitudes and phases of signals from every sensor are different, we can not obtain a better result by just summing the signals from all the sensors. The method of array signal processing $[14,15]$ is to adjust the signals from multiple sensors to have same phase and then summing the signals to increase the amplitude but do not distort the signal. Nevertheless, although after the array signal processing the amplitude of the desired signal is increased, the noises are also amplified. The SNR can not be improved by this way. So how to enhance signal and reduce noise to obtain a higher SNR is the major concern of array signal processing.

To distinguish the speech signal from the background noise, the direction of signal and noise should be defined. The daily life, people who talk to each other are usually face to face. The hearing aids should receive speech signal in this direction. Unfortunately, noises in other direction will interfere with speech signal. By using Griffiths-Jim beamformer, speech signal and noise can be separated. Griffiths-Jim beamformer is a kind of beamformer that using adaptive noise cancellation technique to reduce noise [16]. Signals that collected from multiple sensors can be treated as same desired signal but with different time delay and plus different noise.

The Griffiths-Jim beamformer is shown in Fig. 1. Input signal $I_{k}(n)(k=1 \ldots M)$ are received by multimicrophone array. Let $I_{k}(n)=s_{k}(n)+n_{k}(n)$, here $s_{k}(\mathrm{n})$ is speech signal and $n_{k}(\mathrm{n})$ is noise. Because each microphone received the same speech signal with different time delay, input signal should be adjusted. Beamsteering delays $\tau_{k}(k=I \ldots M)$ can adjust input speech signal $s_{k}(n)$ to have the same time delay. Thus $s_{l}(n)=s_{2}(n)=\cdots=s_{M}(n)=s(n)$ and $x_{k}(n)=s(n)+n_{k}(n)$. The symbol $a_{c k}(k=1 \ldots M)$ is the channel weight. In general, $a_{c 1}=a_{c 2}=\ldots=a_{c M}=1 / M$. Then the desired signal $d(n)$ becomes

$d(n)=\sum_{k=1}^{M} a_{c k} I_{k}=\frac{1}{M} \sum_{k=1}^{M} I_{k}=s+\frac{1}{M}\left(n_{1}+n_{2}+\cdots+n_{k}\right)$

In the Griffiths-Jim beamformer, the objective is to delete the noise term $\left(n_{1}+n_{2}+\cdots+n_{k}\right) / M$. From the adaptive noise cancellation theory, we know that the reference input usually do not have the desired signal. Here we use a matrix processor to separate the desired signal from the reference input. The output of matrix processor $a_{s}^{\prime}$ must meet the requirement of

$a_{s}^{\prime} s(n)=0$, where $S(n)=s(n)\left[\begin{array}{lll}1 & \cdots & 1\end{array}\right]^{r}$.

The matrix $a_{s}^{\prime}$ can remove speech signal from $x_{k}(n)$ and $x_{k}{ }_{k}(n)(k=1 \ldots M)$ contain only noise. The output of adaptive filters are $y_{k}(n)(k=1, \ldots, M-1)$, then

$$
y(n)=\sum_{k=1}^{M-1} y_{k}(n)
$$

Signal $d^{\prime}(n)$ is the signal $d(n)$ after the process of fixed target signal filter. In general, the fixed target signal filter is a time delay. The adaptive filter output, $y(n)$, will equal to $\left(n_{1}+n_{2}+\cdots+n_{k}\right) / M$. Thus,

$$
\begin{aligned}
& e(n)=d^{\prime}(n)-y(n) \\
& =s+\frac{1}{M}\left(n_{1}+n_{2}+\cdots+n_{k}\right)-\frac{1}{M}\left(n_{1}+n_{2}+\cdots+n_{k}\right)=s
\end{aligned}
$$

\subsection{Griffiths-Jim beamformer for the binaural type hearing aids}

For the binaural type hearing aids with two microphones, there are two input signals and $k=1,2$. Because the direction is right ahead, the beam-steering delays $\tau_{k}$ are unnecessary. The channel weight $a_{c l}$ and $a_{c 2}$ are equal to $1 / 2$. The matrix $a_{s}^{\prime}$ is $[1 / 2,-1 / 2]$. There is only one adaptive filter in the binaural type hearing-aid system. The fixed target signal filter is a $D$ clocks delay, and $D$ is the order of the adaptive filter. The Griffiths-Jim beamformer can then be simplified to as shown in Fig. 2 [17]. If $x_{l}(n)=s_{/}(n)+n_{/}(n)$ and 
$x_{2}(n)=s_{2}(n)+n_{2}(n)$. Because what we desired is the sound from right ahead, $s(n)=s_{/}(n)=s_{2}(n)$, and so that

$$
\left(x_{1}(n)+x_{2}(n)\right) / 2=s(n)+\left(n_{1}(n)+n_{2}(n)\right) / 2,
$$

and

$$
\left(x_{1}(n)-x_{2}(n)\right) / 2=\left(n_{1}(n)-n_{2}(n)\right) / 2=x(n) .
$$

Where $x(n)$ is the input signal to the adaptive filter. $d(n)$ is a $D$ clocks delayed version of $\left(x_{1}(n)+x_{2}(n)\right) / 2$.

$$
d(n)=s(n-D)+\left(n_{1}(n-D)+n_{2}(n-D)\right) / 2
$$

Finally, the output of Griffiths-Jim beamformer, $e(n)$, will equal to the speech signal, $s(n)$. The whole system is illustrated in Fig. 2.

\subsection{Subband adaptive filtering}

There are two types of multirate filter banks: uniform filter bank and nonuniform filter bank. Uniform filter bank is a special case of nonuniform filter bank. The request for computation of single band adaptive filter algorithm is less than full band adaptive filter algorithm when using the nonuniform filter banks. The other advantages of multirate processing are to provide less computational and storage requirements as well as lower filter order. This makes it very suitable for digital signal processing. For audiogram applications, nonuniform frequency is split based on octave frequency bands of human ears. The frequency response of the decimation filter banks can be individually adjustable in each octave band [18].

Multirate filter banks can be constructed from two-channel filter banks. We will first explain the ba- sic principles of two-channel filter bank and perfect reconstruction (PR) condition. As shown in Fig. 3, two-channel filter bank is constructed from analysis filters, downsampling operation, upsampling operation, and synthesis filters. Analysis filters and synthesis filters of two-channel filter bank are constructed from a high-frequency and a low-frequency filters respectively. Analysis filters decompose the signal into highfrequency and low-frequency bands. Synthesis filters will recompose the original signal. Several signal processing schemes, such as data compressing and adaptive filtering, can be added to the frequency change operand between analysis filters and synthesis filters.

If $H_{0}(z)$ is the low-pass filter, $H_{1}(z)$ is the complemented high-pass filter. The Z-transforms of the decimated signals in Fig. 3 are the output of analysis filters,

$$
\begin{aligned}
& x_{1}(z)=X(z) H_{0}(z) \\
& x_{2}(z)=X(z) H_{1}(z) \\
& y_{1}(z)=\frac{1}{2} X\left(z^{1 / 2}\right) H_{0}\left(z^{1 / 2}\right)+\frac{1}{2} X\left(-z^{1 / 2}\right) H_{0}\left(-z^{1 / 2}\right)
\end{aligned}
$$

$$
y_{2}(z)=\frac{1}{2} X\left(z^{1 / 2}\right) H_{0}\left(z^{1 / 2}\right)+\frac{1}{2} X\left(-z^{1 / 2}\right) H_{1}\left(-z^{1 / 2}\right)
$$

In a similar way, $G_{0}(z)$ is the synthesis lowpass filter, $G_{1}(z)$ is the high-pass filter, the Ztransform is

$$
\hat{X}(z)=G_{0}(z) y_{1}\left(z^{2}\right)+G_{1}(z) y_{2}\left(z^{2}\right)
$$

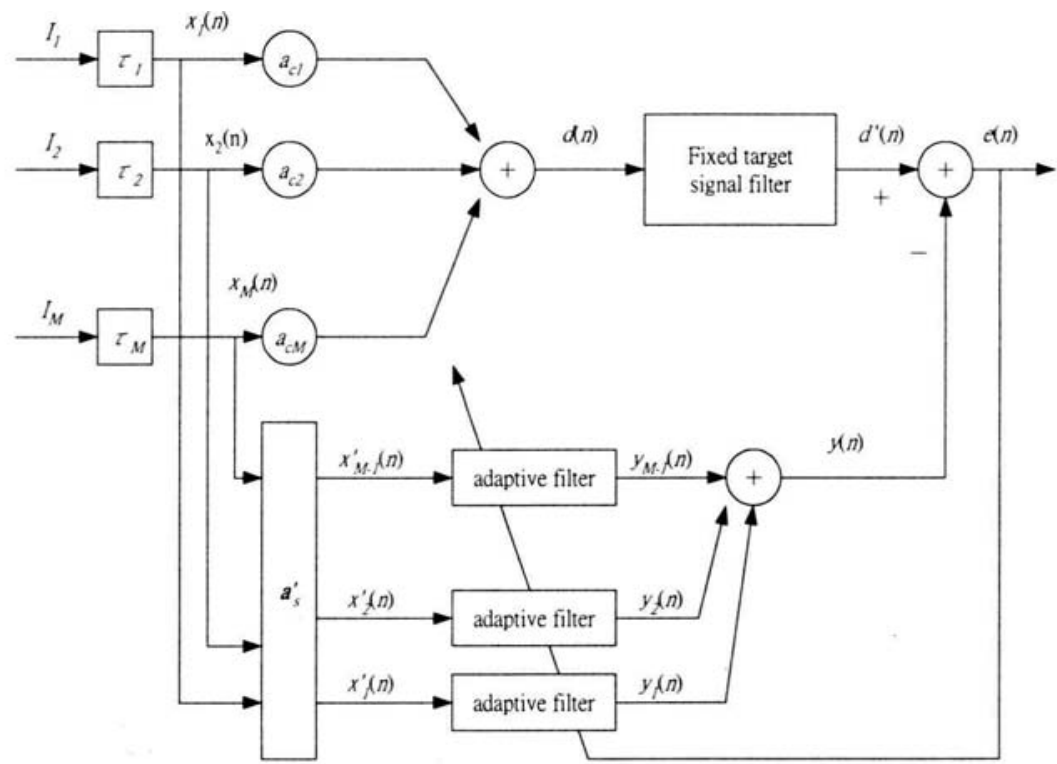

Fig. 1. Griffiths-Jim beamformer. 


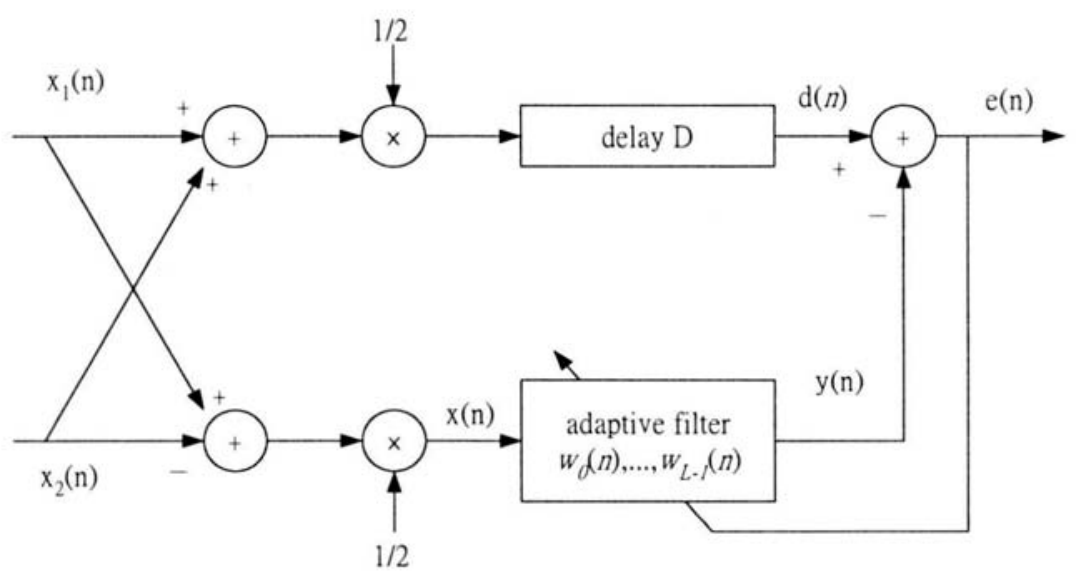

Fig. 2 Binaural type Griffiths-Jim beamformer.

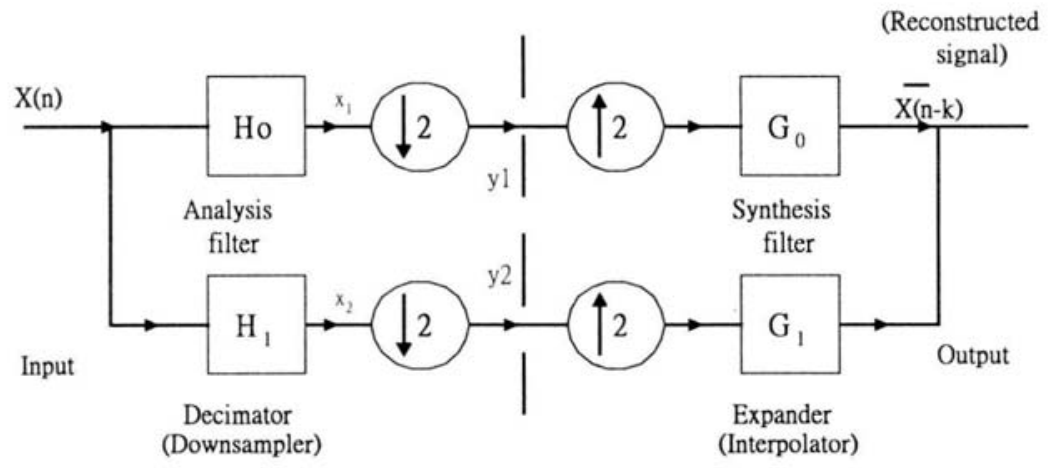

Fig. 3. Structure of multirate filter bank.

reconstrucled signal

$$
\begin{aligned}
\dot{X}(z)= & \left.\frac{1}{2} \mid G_{0}(z) H_{0}(z)+G_{1}(z) H_{1}(z)\right] X(z) \\
& +\frac{1}{2}\left[G_{0}(z) H_{0}(-z)+G_{1}(z) H_{1}(-z)\right] X(-z) \\
& =F_{0}(z) X(z)+F_{1}(z) X(-z),
\end{aligned}
$$

where

$$
F_{11}=\frac{1}{2}\left[G_{11}(z) H_{0}(z)+G_{1}(z) H_{1}(z)\right],
$$

and

$$
F_{1}=\frac{1}{2}\left[G_{0}(z) H_{10}(-z)+G_{1}(z) H_{1}(-z)\right] .
$$

From the above equations we can see that $\hat{X}(z)$ suffers from thrce fundamental errors: aliasing, amplitude distortion, and phase distortion. The aliasing can be removed if we let $F_{1}=0$ in (6). That's because $X(-z)$ is the noise in the high-frequency band (between $\pi$ and $2 \pi$ ) that is folded to the lowfrequency band of the system. This term has the highest priority when design a filter, because this term has the most significant effect to the SNR. If $F_{1}=0$, we can obtain a set of relations, i.e., $G_{0}(z)=H_{j}(-z)$ and $G_{1}(z)=-H_{0}(-z)$. The other distortions are determined by $F_{0}$. If we let $H_{0}(z)=H(z)$ and $H_{1}(z)=H(-z)$. where $H(z)$ is a low-pass filter, the final result will becomes $2 z{ }^{4}$. That is, the output of $\hat{X}(n)$ is the $k$ delays version of the original signal. Then we can obtain a perfect reconstruction condition, i.e., solution of quadrature mirror filter (QMF). The other filters that frequently used are conjugate QMF, half-band filter, cosine modulated filter and over-sampled filter bank [19].

We can further decompose the frequency bands by adding another two-channel filter bank to the output of the low-pass filter of the preceding two-channel filter bank. Then we can obtain nonuniform filter banks that are decomposed based on octave frequency bands. We can do this recursively to further decompose the spectra (Fig. 4). Since the separation is from high frequency to low frequency, lower-frequency bands will have higher resolution. In a similar way, we can perform the separations from low frequency to high fre- 


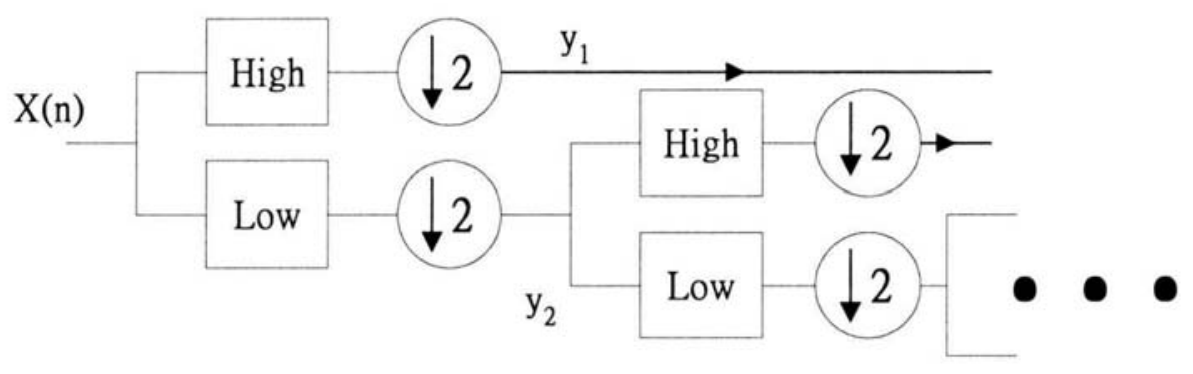

Fig. 4. Tree structured multirate analysis filter bank.

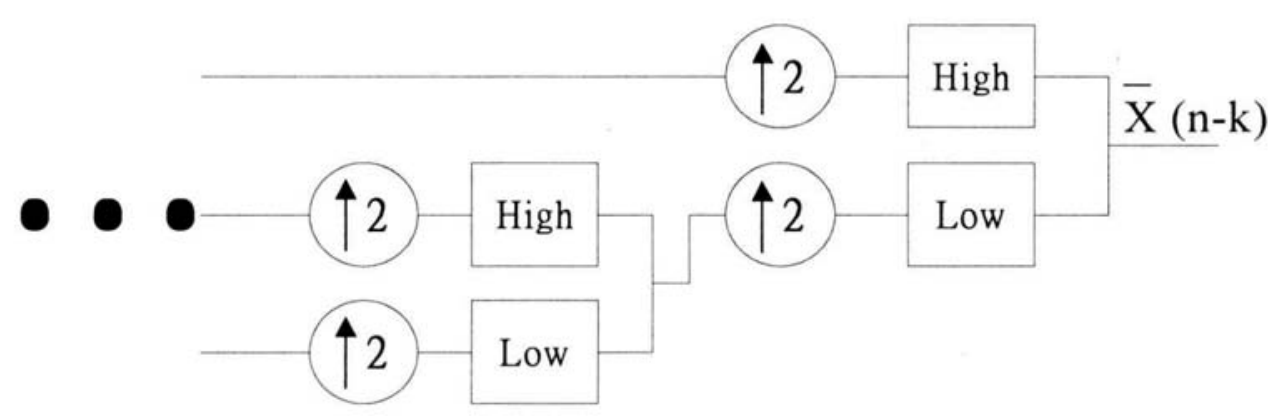

Fig. 5. Tree structured multirate synthesis filter bank.

quency and obtain higher resolution for higherfrequency bands. We can use different tree structures to get the best results for different conditions. As shown in Fig. 5, when using the tree structured twochannel filter banks to synthesis the signal, we can adjust the amplitude of the signal in the synthesis filter. In this way, we can enhance the bands that need to be emphasized. Furthermore, if we add filters to the outputs of the preceding high-pass and low-pass filters, then we can get multiple bands for low- and highfrequency bands that are decomposed from the input signal.

Subband adaptive filter is a combination of adaptive filtering and multirate filter banks algorithms. As shown in Fig. 6, the system split the inputs into two parts, that is input signal, $d(n)$, and noise reference input, $x(n)$. The two inputs then pass through the tree structured analysis filters of themselves. The adaptive noise cancellation algorithm is applied to each band. Here $\left(Y_{0}, Y_{1}, Y_{2}, \ldots\right)$ is the signal $d(n)$ after the process of tree structured analysis filters, and $\left(\hat{Y}_{0}, \hat{Y}_{1}, \hat{Y}_{2}, \ldots\right)$ is the signal of $x(n)$ after the process of tree structured analysis filters. The errors $\left(e_{0}, e_{1}, e_{2}, \ldots\right)$ will pass through the tree structured synthesis filters and recompose the original signal $[6,7,8]$.

\section{HARDWARE SYSTEM AND SOFTWARE DESIGN}

\subsection{System structure}

Fig. 7 shows the structure of a binaural type digital hearing-aid system. The system is implemented and tested on the Texas Instruments TMS320VC5402 digital signal processor DSK with dual microphones and speakers. Because there is only one channel codec for microphone and speaker on the MS320VC5402 DSK (another channel is for DAA applications), it has been extended to two channels of ADC input and one channel DAC output. Two omni-directional microphones were used to sense the speech signal and the surrounding noise. Microphone preamplifier, anti-aliasing filter, and mixer filter are also applied to this system.

The signals of the two microphones were digitized at a sampling frequency of $22.05 \mathrm{kHz}$ by two $12-$ bit analog-to-digital converters (SPT574). The DSP executes algorithms to enhance the right ahead speech signal and reduce background noise to improve SNR. The processed digital output signals from the DSP were reconstructed with 12-bit digital-to-analog converter (AD565) and filtered by a low-pass filter. The final improved signals are displayed from the oscilloscope. We also stored the signal data and used MAT$\mathrm{LAB}^{\mathrm{TM}}$ to analyze and display the results. 


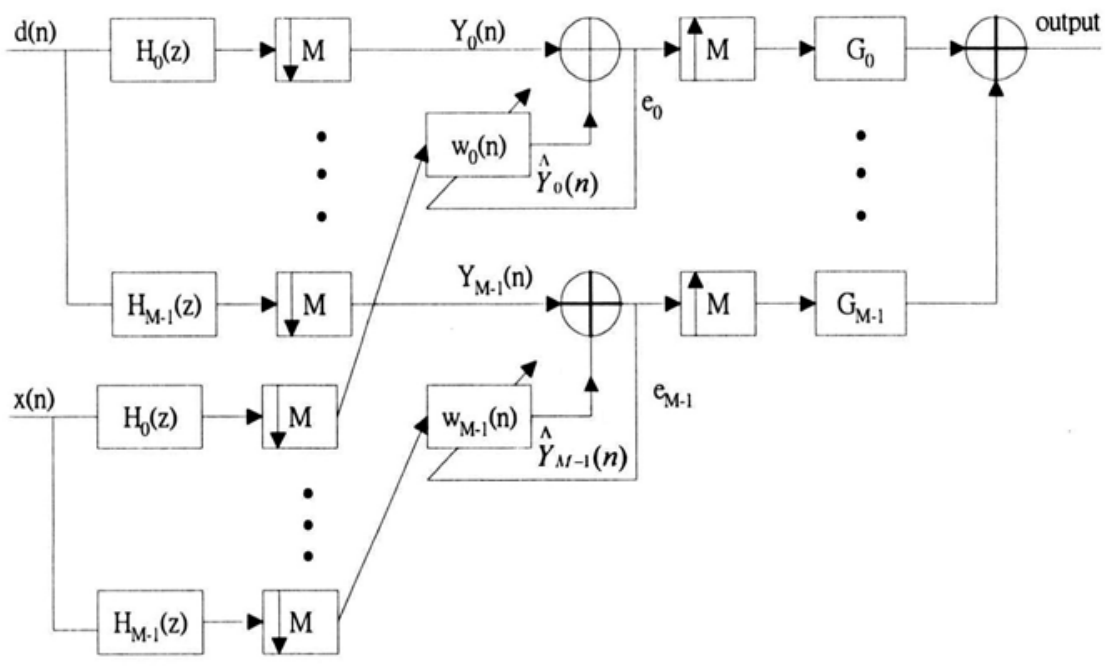

Fig. 6. Subband adaptive filtering.

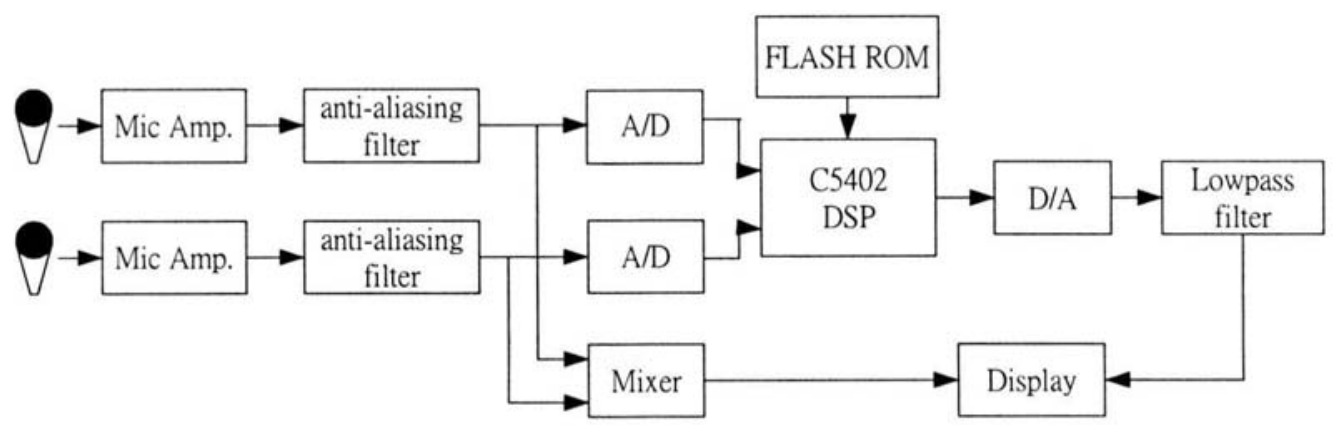

Fig. 7. The structure of digital hearing aids.

\subsection{Software development}

The algorithm was coded by using the TI C5000 assembly language. This is to make sure that the developed system has efficiently utilized the highest performance of the hardware. The TI TMS320C5402 DSP has pipeline structure that increases the performance of program execution. NLMS algorithm has the ability to achieve the requirement of high order coefficients but do not become a burden to the hardware system. And the subband adaptive filter can reduce the calculations of system. When using the analysis filter and synthesis filter, one must be careful to avoid the aliasing and amplitude distortion. We can use different cutoff frequency to get the best performance. For the $100 \mathrm{MHz}$ DSP, $22.05 \mathrm{kHz}$ sampling frequency indicates that the maximum period to execute the algorithm is about 5000 clocks. Figure 8 shows the adaptive beamformer system that adopts subband filtering with NLMS algorithm to achieve wider frequency band.

\section{EXPERIMENTS AND RESULTS}

The performance of this system is illustrated and tested by using simulated and real-world noises. A simulated human head was used to test the capability of the system. We will first evaluate the direction discriminating ability of the system. In noise cancellation test, we have tried two situations. The first case used an AM signal to represent the speech signal and a running hair dryer as the noise source. FLS (fast least mean square) and NLMS (normalized least mean square) adaptive filtering algorithms with and without using subband method were used to test system. The second test used a practical human speech as source signal and a sine wave as the noise. The time-domain and frequency-domain results of both cases were recorded for comparison.

\subsection{Direction test}




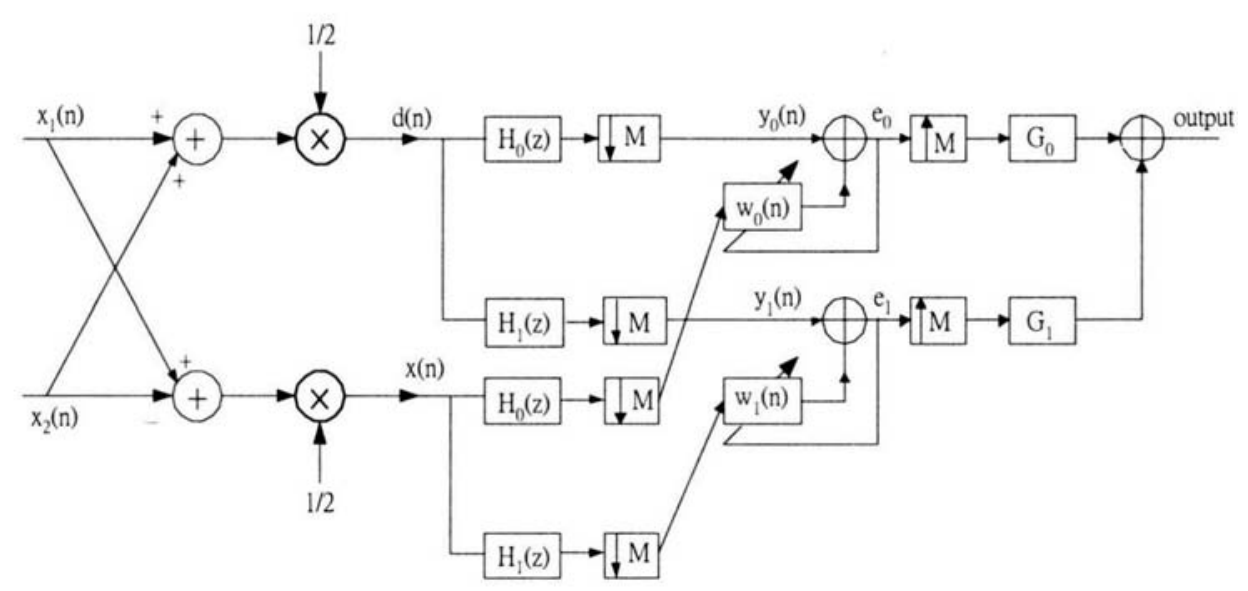

Fig. 8. Adaptive beamformer system adopts subband filtering with NLMS algorithm

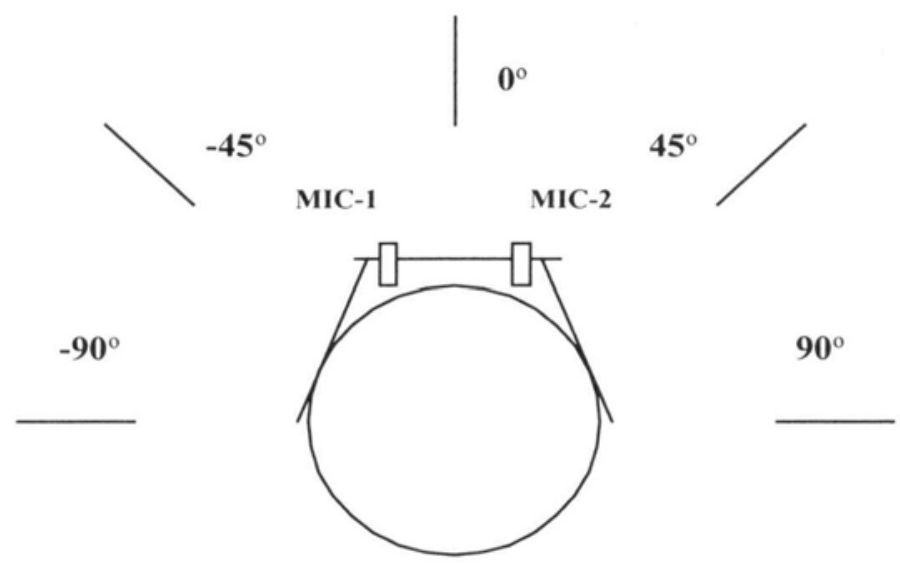

Fig. 9 Simulated human head and the locations of microphones.

A $25-\mathrm{cm}$ diameter expandable polystyrene ball was used to simulate the human head. We used two omni-directional microphones to sense the speech signal and the background noise. The microphones were fixed on eyeglasses that put on the head. The distance between two microphones is $13 \mathrm{~cm}$. This distance is near to the width of practical human eyeglasses. The simulated human head was put in the center. Straight ahead of the head is defined as zero degree. Right hand side is 90 degrees and left hand side is -90 degrees (Fig. 9).

Signals that collected from the two microphones were first processed using anti-aliasing filters. We recorded both the summation results from the two microphones and the signal after the processing of DSP. The results will be showed and compared. Here we used $1-\mathrm{kHz}$ frequency modulated (FM) wave to test the system. Signal source was moved around the head to test the noise reduction. We measured the signal level when the signal source was moved around the head from -90 degrees to 90 degrees with 10 degrees step. The results for the cases when the cutoff frequencies are $4.5 \mathrm{kHz}$ and $3.7 \mathrm{kHz}$ are shown in Fig. 10. The detected strongest signal is in the zero degree. We defined this result as $0 \mathrm{~dB}$. As shown, noise in every location of the other directions has been reduced at least $10 \mathrm{~dB}$.

\subsection{Noise reduction experiments}

In the following tests, we will show that the system can reserve speech signal in zero degree and reduce noise from other directions. Adaptive beamformer adopts NLMS algorithm with and without using the subband method and FLS algorithm alone were used to test system. Simulated signal source with a frequency of $1-\mathrm{kHz}$ AM wave was placed in front of the head and a running hair dryer as the noise source was put in the 45 degrees direction with a distance at $45 \mathrm{~cm}$. The sampling frequency is $22.05 \mathrm{kHz}$ for all the cases shown. The time-domain and frequencydomain results before and after the processing of DSP were recorded and compared as shown in Fig. 11-13. 


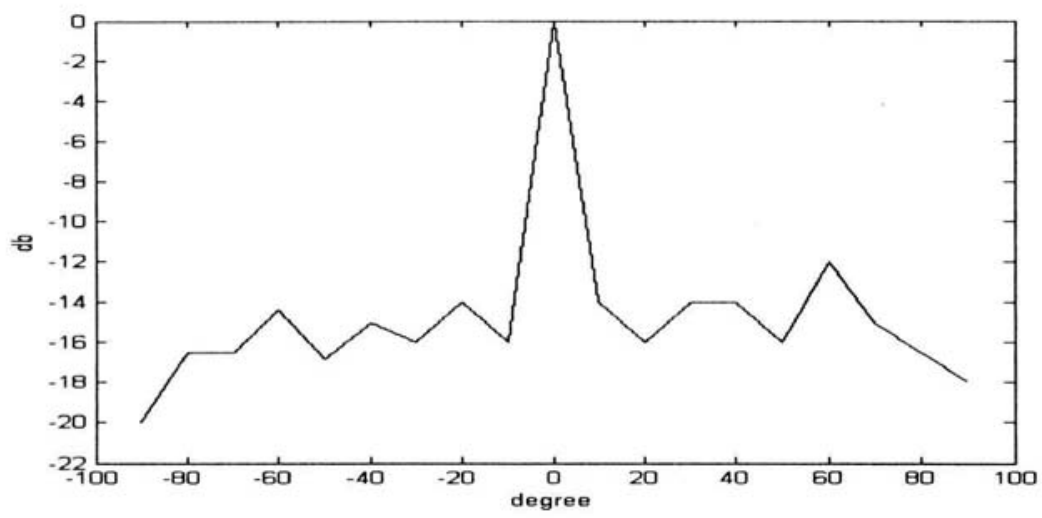

(a)

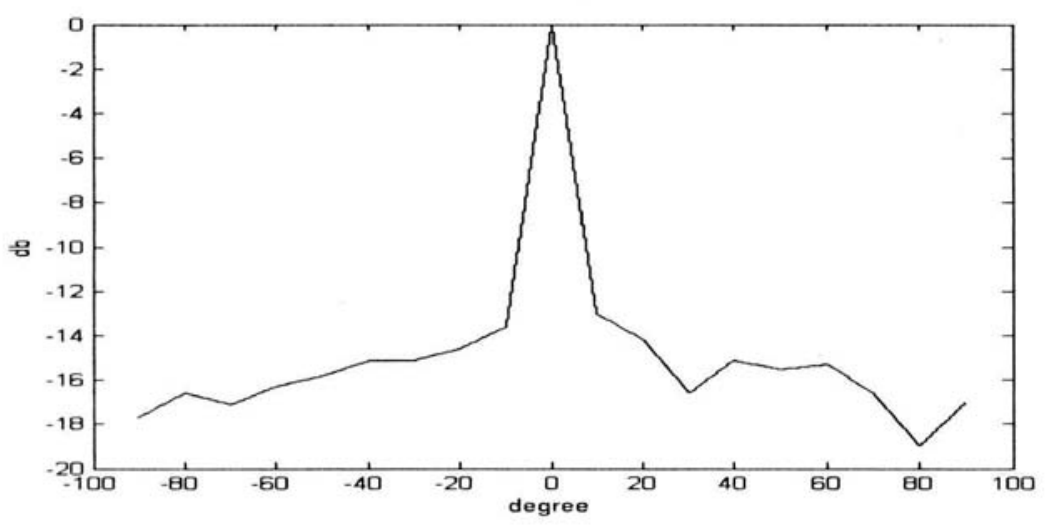

(b)

Fig. 10. Noise reduction on directions from -90 to 90 degrees (cutoff frequency @ a. $4.5 \mathrm{kHz} ;$ b. $3.7 \mathrm{kHz}$ )

Fig. 11 is the case for the NLMS algorithm without using the subband method. The upper two sub-graphs are the time-domain and frequency-domain of the original signals (i.e. AM signal plus noise) before processing. The lower two sub-graphs are the time-domain and frequency-domain of the signals after processing. The SNR has 2.5-dB improvement. Fig. 12 shows another case for FLS algorithm without using the subband method. The SNR has 2.1-dB improvement before and after processing. Fig. 13 is the case for NLMS algorithm with using the subband method. The cut-off frequency is $5 \mathrm{kHz}$. The SNR has $3.05-\mathrm{dB}$ improvement before and after processing.

The second testing used a practical human speech as signal and a $1-\mathrm{kHz}$ pure sine wave as the noise. The locations and directions of both signal and noise are same as the previous case. The sampling frequency is $22.05 \mathrm{kHz}$ for all the cases shown. The time-domain and frequency-domain results before and after processing of the DSP were recorded and compared as shown in Fig. 14-16. Fig. 14 is the case for the NLMS algo- rithm without using the subband method. The SNR has 13.8-dB improvement before and after processing. Fig. 15 is the case for the FLS algorithm without using the subband method. The SNR has 10.2-dB improvement before and after processing. Fig. 16 shows the case for NLMS algorithm with using the subband method. The cut-off frequency is $5 \mathrm{kHz}$. The SNR has $15.5-\mathrm{dB}$ improvement before and after processing.

\section{DISCUSSION}

The results show that the developed system can reserve the right ahead speech signal and substantially reject noises from other directions. Noise has been reduced at least $10 \mathrm{~dB}$ in the direction tests. In this test, we assumed that the desired signal was came from the zero degree (i.e. right ahead). It is not a problem to rearrange the system when signal from other direction is desired. That means one can enhance the system to reserve the signal from any specific direction and reduce 

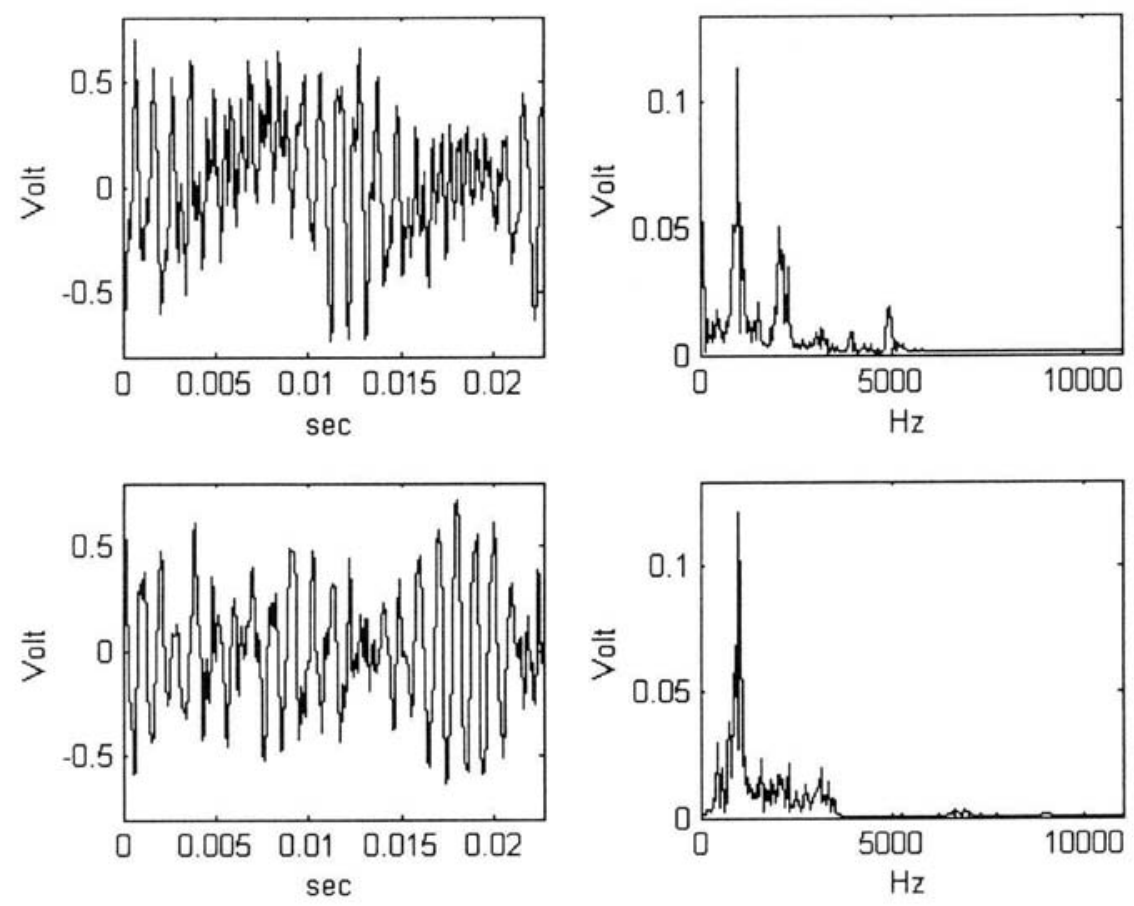

Fig. 11. Adaptive beamformer adopts NLMS algorithm without using the subband method.
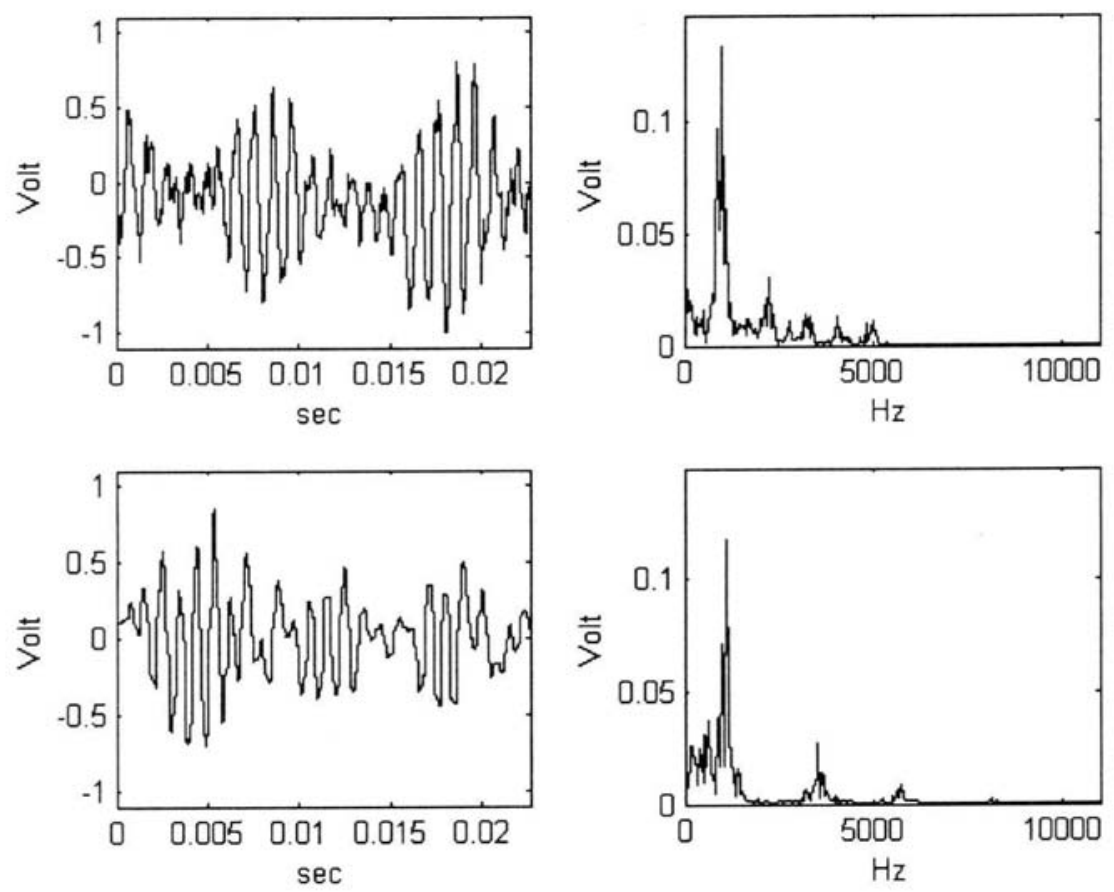

Fig. 12. Adaptive beamformer adopts FLS algorithm without using the subband method. 

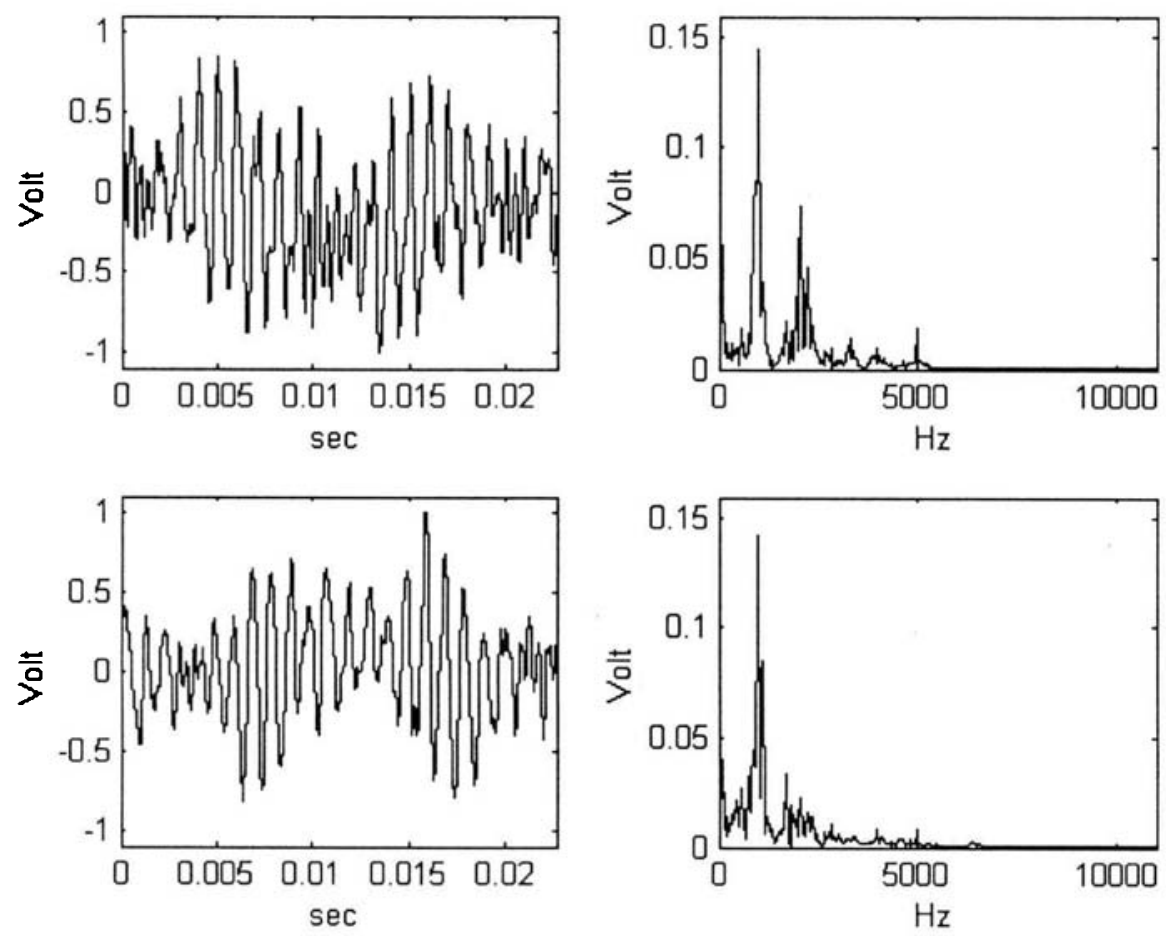

Fig. 13. Adaptive beamformer adopts NLMS algorithm and the subband method.
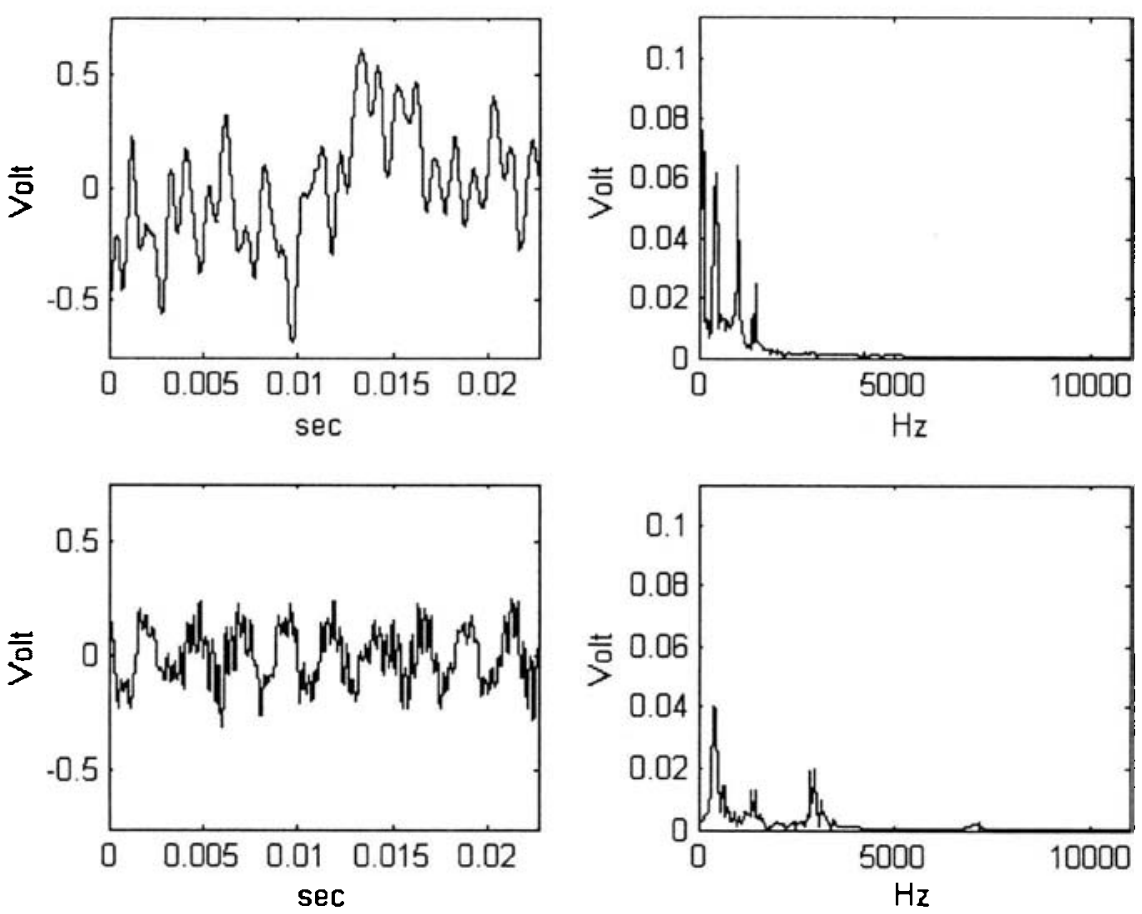

Fig. 14. Adaptive beamformer adopts NLMS algorithm without using the subband method. 

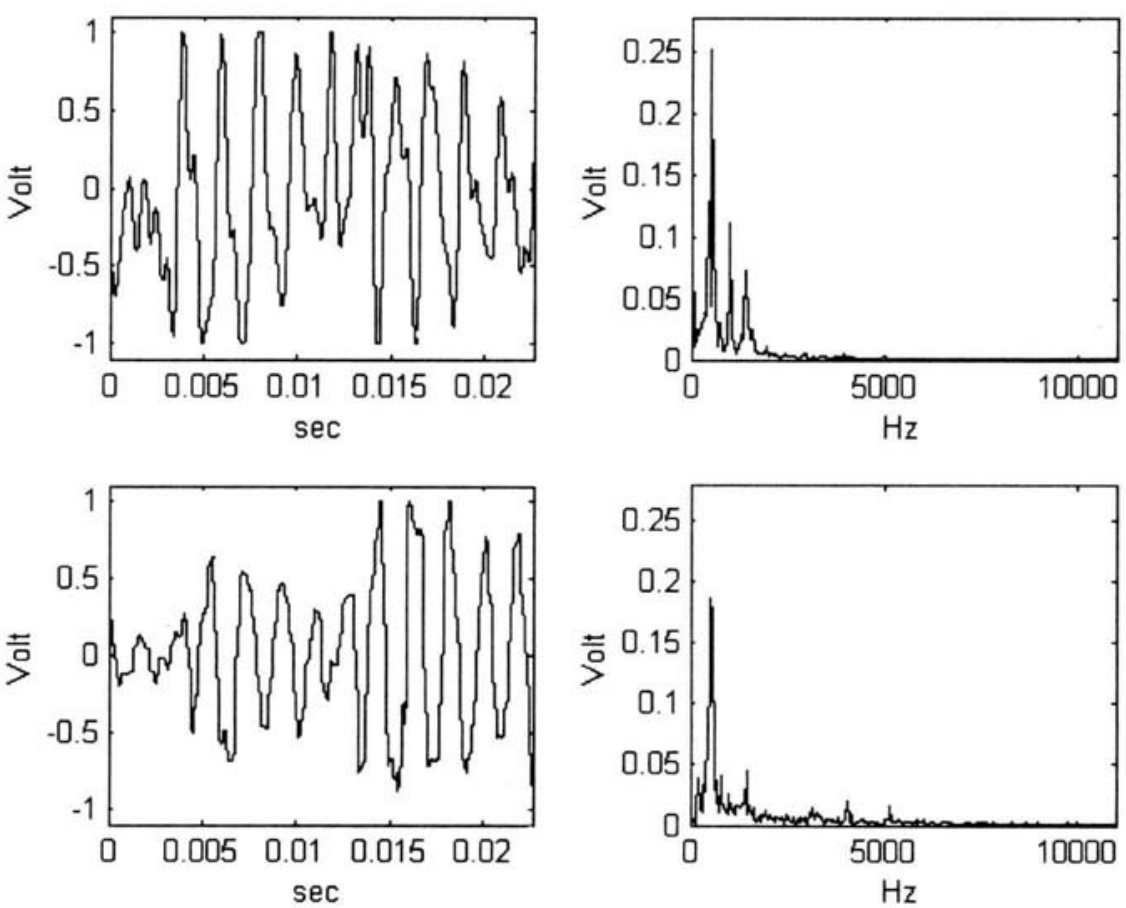

Fig. 15. Adaptive beamformer adopts FLS algorithm without using the subband method.
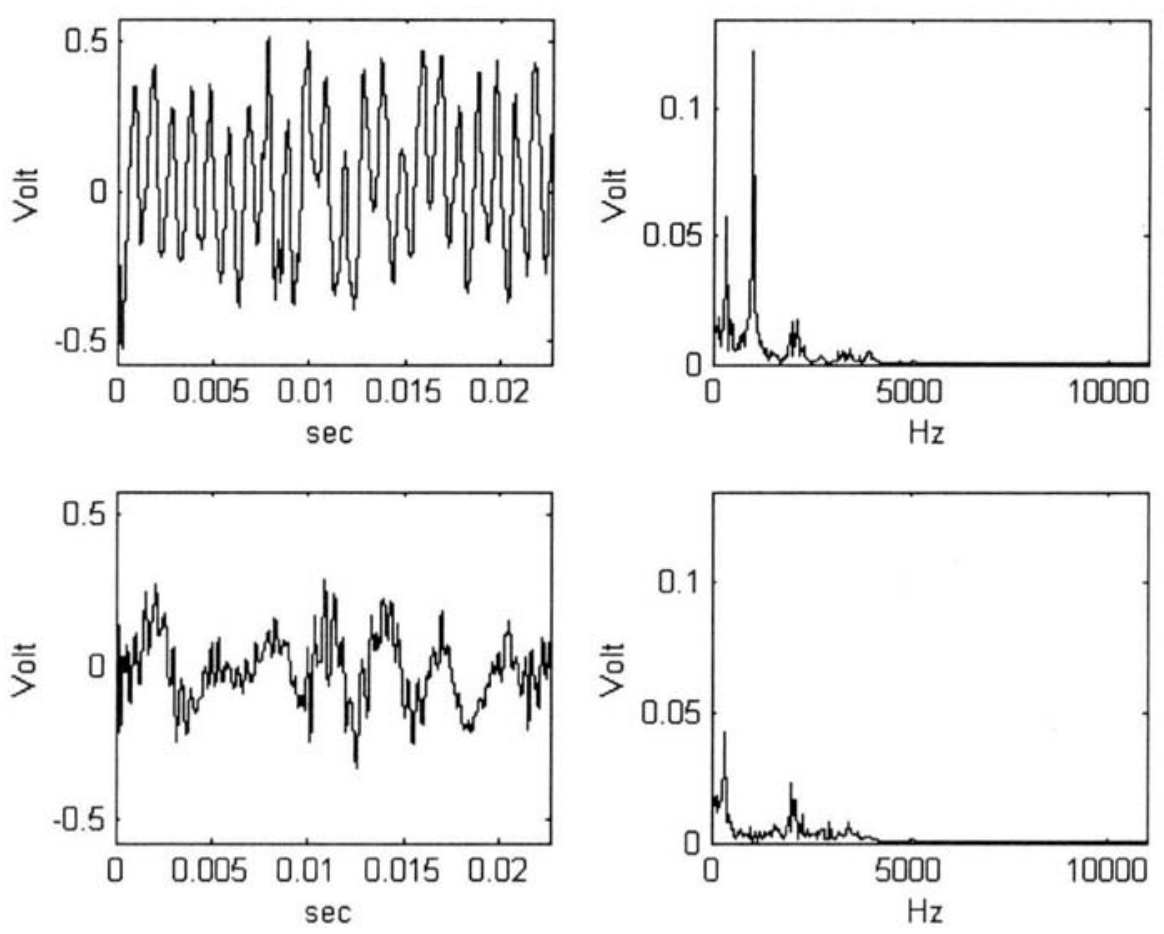

Fig. 16. Adaptive beamformer adopts NLMS algorithm and the subband method. 
the sounds from other directions.

For the first case that using simulated signal source with a frequency of $1-\mathrm{kHz}$ AM wave and a running hair dryer as the noise source, the improvement in SNR is from $2.1 \mathrm{~dB}$ to $3.05 \mathrm{~dB}$. It is not a great improvement. This is because the noise from the running hair dryer is a near-random broadband noise. The DSP can not exactly trace the noise.

The second testing used a practical human speech as signal and a $1-\mathrm{kHz}$ sine wave as the noise. The improvement in SNR is from $10.2 \mathrm{~dB}$ to $15.5 \mathrm{~dB}$. This result is much better than the first case. We interpret this phenomenon is because the noise used in this case is a narrowband noise (pure sine wave). The DSP is much easier to trace the noise. We then conclude that the developed system can adequately reduce narrowband noise and slightly reduce broadband random noise.

\section{REFERENCES}

1. Widrow B: Adaptive Signal Processing. PrenticeHall. 1985.

2. Haykin S: Adaptive Filter Theory. Prentice-Hall. 1991.

3. Sen MK, Dennis RM: Active Noise Control Systems. John Wiley \& Sons, New York. 1996.

4. Greenberg JE, Zurek PM: Evaluation of an Adaptive Beamforming Method for Hearing Aids. Journal of the Acoustical Society of America. 1992; 91: $1662-1676$.

5. Maurice G. B: Adaptive Digital Filter and Signal Analysis. Marcel Dekker Inc. 1988.

6. Sandeep PS, Reddy VU: A New Approach to Subband Adaptive Filtering. IEEE Transactions on Signal Processing. 1999; 47: 655-664.

7. Harteneck M, Stewart RW: A Subband Adaptive
Filter. IEE Colloquium on Adaptive Signal Processing for Mobile Communication Systems.1997; $1 / 1-1 / 6$.

8. Sakai H, Hirayama N: Analysis of Subband Adaptive Filters. Acoustics, Speech, and Signal Processing, ICASSP-96 Conference Proceedings. 1996; $1303-1306$.

9. Vaidyanathan PP: Multirate Systems and Filter Banks. Prentice Hall Inc, Englewood Cliffs. 1993.

10. Crochiere RE, Rabiner LR: Multirate Digital Signal Processing. Prentice-Hall Inc, Englewood Cliffs. 1983.

11. Fliege NJ: Multirate Digital Signal Processing. John Wiley \& Sons. 1994.

12. Strang G, Nguyen T: Wavelets and Filter Bank. Wellesley-Cambridge Press. 1996.

13. Li M, McAllister HG, Black ND: Multirate Modeling of Human Ear Frequency Resolution for Hearing Aids. Digital Signal Processing Workshop Proceedings, IEEE.1996; 157-160.

14.Johnson DH, Dudgeon DE: Array Signal Processing. Prentice Hall. 1993.

15.Julie EG: Improved design of microphone-array hearing aids. Ph.D. dissertation, Harvard-MIT Division of Health Sciences and Technology, Cambridge, MA. 1994.

16. Griffiths LJ, Jim CW: An Alternative to Linearly Constrained Adaptive Beamforming. IEEE Transaction on Antennas and Propagation. 1982.

17. Fischer S, Simmer KU: Beamforming Microphone Arrays for Speech Acquisition in Noisy Environments. Speech communication. 1996; 20: 215-227.

18.Zolzer U: Digital Audio Signal Processing. John Wiley \& Sons. 1998.

19. Khalid S: Introduction to Data Compression. Morgan Kaufmann. 2000. 